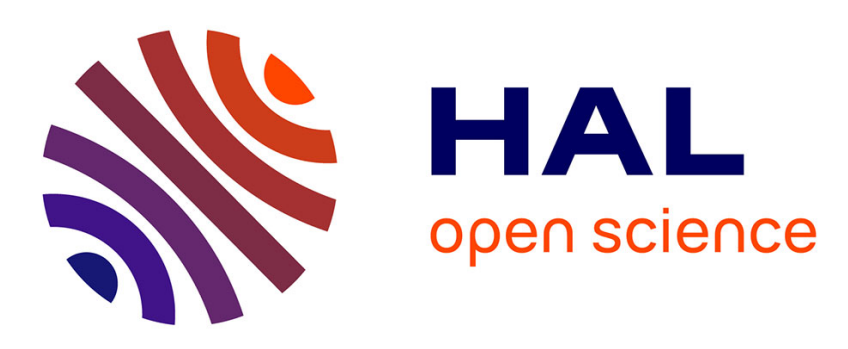

\title{
Nash Equilibria for multi-agent network flow with controllable capacities
}

\author{
Nadia Chaabane, Cyril Briand, Marie-José Huguet
}

\section{To cite this version:}

Nadia Chaabane, Cyril Briand, Marie-José Huguet. Nash Equilibria for multi-agent network flow with controllable capacities. Communications in Computer and Information Science, 2015, Operations Research and Enterprise Systems, 509, pp.47-62. 10.1007/978-3-319-17509-6_4 . hal-01166010

\section{HAL Id: hal-01166010 https://hal.science/hal-01166010}

Submitted on 21 Jun 2015

HAL is a multi-disciplinary open access archive for the deposit and dissemination of scientific research documents, whether they are published or not. The documents may come from teaching and research institutions in France or abroad, or from public or private research centers.
L'archive ouverte pluridisciplinaire HAL, est destinée au dépôt et à la diffusion de documents scientifiques de niveau recherche, publiés ou non, émanant des établissements d'enseignement et de recherche français ou étrangers, des laboratoires publics ou privés. 


\title{
Nash Equilibria for multi-agent network flow with controllable capacities
}

\author{
Nadia Chaabane ${ }^{1,2}$, Cyril Briand ${ }^{1,3}$, and Marie-José Huguet ${ }^{1,2}$ \\ 1 CNRS, LAAS, 7 avenue du colonel Roche, F-31400 Toulouse, France \\ 2 Univ de Toulouse, INSA, LAAS, F-31400 Toulouse, France \\ 3 Univ de Toulouse, UPS, LAAS, F-31400 Toulouse, France
}

\begin{abstract}
In this work, a multi-agent network flow problem is addressed where a set of transportation-agents can control the capacities of a set of elementary routes A third-party agent, a customer, is interesting in maximizing the product flow transshipped from a source to a sink node through the network and offers a reward that is proportional to the flow value the transportation agents manage to provide. This problem can be viewed as a Multi-Agent Minimum-Cost Maximum-Flow Problem where the focus is put on finding stable strategies (i.e., Nash Equilibria) such that no transportation-agent has any incentive to modify its behavior. We show how such an equilibrium can be characterized by means of augmenting or decreasing paths in a reduced network. We also discuss the problem of finding a Nash Equilibrium that maximizes the flow and prove its NP-Hardness.
\end{abstract}

Keywords: Multi-Agent Network flow, Nash Equilibria, Complexity, MinCost Max-Flow.

\section{Introduction}

Multi-agent network games have become a promising interdisciplinary research area with important links to many application fields such as transportation networks, supply chain management, web services, production management, etc [1], [2]. In these applicative areas, decision processes often involve several agents, each one having its own autonomy, its own objectives and its own constraints. These actors, often referred to as agents, need to cooperate together to fulfill a global (social) goal, provided their own objective is also satisfied. This paper stands at the crossroads of two disciplines, namely multi-agent systems and social networks. A network flow that involves a set of agents, each one being in charge of a part of the network, is considered in this paper, where every agent is able to control the capacities of its arcs at a given cost. We address the problem of finding a Nash equilibrium that maximizes the flow transported through the network. A lot of features used in this work are inspired by the Multi-Agent Project Scheduling (MAPS), as presented in [3], especially the payment scheme: the outcome of an agent depends on its own strategy and on the satisfaction of a customer, which depends on the flow circulating in the network. This paper 
mainly discusses the complexity of finding a Nash Equilibrium that maximizes the flow in the network.

To the best of our knowledge, the research presented here is an original way of presenting a transportation problem using multi-agent network flow with controllable arcs capacities. One important application is the expansion of transportation network capacity (railway, roads, pipelines, etc.) to meet current peak demand or to absorb future increase in the transportation demands. Therefore, it is possible to increase the capacity of the network using two solutions: either increase the capacity of one or many existing arcs or installing a new arc between two nodes.

A natural problem in many network applications is where to increase arc capacities so that to increase the overall flow in the network at minimum cost. There exists substantial research on capacity expansion (or capacity planning) problems in different domains, such as manufacturing [4], electric utilities [5], telecommunications [6], inventory management [7], and transportation [8], [9] and [10].

As regards to social networks, the prediction of agents' behavior is of interest. Several papers focus on games associated with various forms of networks, see [11] for an overview. In a recent work, Apt and Markakis (2011) studied the complexity of finding a Nash Equilibrium for the multi-agent social networks with multiple products, in which the agents, influenced by their neighbors, can choose one out of several alternatives [12]. In [13], a cooperative network flow game is considered, where an external party gives an additional payment to the coalition, which may stabilize the game if the payment is sufficiently high. They study the Cost of Stability (CoS) in threshold network flow games where each agent controls an edge in the network.

A decade ago, some researchers have paid attention to a particular multi-agent network problem: the Multi-Agent Project Scheduling problem (MAPS) that describes a project scheduling environment in which the activities of the project network are partitioned among a set of agents. In the seminal work of Evaristo and Fenema (1999) [14], a special framework for distributed projects is proposed, with costs and rewards shared among agents. In an earliest work [15], the authors considered a MAPS problem where each agent can control the duration of its activities at a given cost. The project activities and precedence constraints are classically modeled with an activity-on-arc graph. A reward is offered to the agents when they manage to finish the project earlier than expected, as proposed in [16]. It has been demonstrated in [3] and [17] that finding a Nash equilibrium minimizing the project makespan is NP-hard in the strong sense. Moreover, using the concepts of an increasing and decreasing cut defined in [18] and the duality between maximum flow and minimum cut problems, Briand et al. (2012b) proposed an efficient integer linear program formulation for this problem [19].

The paper is organized as follows: Section 2 defines formally the Multi-Agent Minimum-Cost Maximum-Flow problem and introduces some important notations. Thereafter, Section 3 introduces the requirements for agents' strategies and presents some important properties. In Section 4, some useful particular 
cases are considered, namely the single agent, the general multi-agent and the special multi-agent cases. Section 5 focuses on the complexity of some decision problems. Finally, conclusions and future directions are drawn in Section 6.

\section{Problem Statement and Notations}

We focus on a Minimum-Cost Maximum-Flow problem in a Multi-Agent context. This problem will be further referred to as MA-MCMF. In this work, a major assumption is that arc capacities are controlled by some agents, called transportation-agents, each arc being assigned to a specific agent.

As in [16], we assume that a customer-agent gives a reward proportional to the flow that circulates inside the network. This reward is shared among transportationagents according to some ratios collectively agreed during the network design phase [20]. Considering a network flow with limited arc capacities, this problem consists in sending a maximum amount of products (for the customer) from a source node to a sink node, at minimum cost (for the transportation-agents).

\subsection{Problem Definition}

The MA-MCMF problem is defined by a tuple $\langle G, \mathcal{A}, \underline{Q}, \bar{Q}, C, \pi, W>$ where:

- $G=(V, E)$ is a network flow. $V$ is the set of nodes, $s, t \in V$ being the source and the sink nodes of the network flow $G$, respectively. $E$ is the set of arcs, each one having its capacity and receiving a flow. An arc $e$ from node $i$ to node $j$ is denoted by $e=(i, j)$.

- $\mathcal{A}$ is a set of $m$ transportation-agents: $\mathcal{A}=\left\{A_{1}, \ldots, A_{u}, \ldots, A_{m}\right\}$. Arcs are distributed among the agents. An agent $A_{u}$ owns a set of $m_{u}$ arcs, denoted $E_{u}$. Each $\operatorname{arc}(i, j)$ belongs to exactly one transportation-agent (i.e., $E_{u} \cap$ $E_{v}=\emptyset$ for each agent's pair $\left(A_{u}, A_{v}\right) \in \mathcal{A}^{2}$ such that $\left.u \neq v\right)$.

- $\underline{Q}$ (resp. $\bar{Q}$ ) represents the vector of normal (resp. maximum) capacity for each arc $(i, j) \in E: \underline{Q}=\left(\underline{q}_{i, j}\right)_{(i, j) \in E}$ and $\bar{Q}=\left(\bar{q}_{i, j}\right)_{(i, j) \in E}$.

- $C=\left(c_{i, j}\right)_{(i, j) \in E}$ is the vector of costs where $c_{i, j}$ is the unitary cost incurred by agent $A_{u}$, for increasing $q_{i, j}$ by one unit. The vector $C_{u}$ denotes the cost vector incurred when augmenting the capacity of its arcs.

$-\pi$ refers to the reward given by the final customer. This reward is proportional to the flow that circulate from $s$ to $t$.

- $W=\left\{w_{u}\right\}$ defines the sharing policy of rewards among the agents. The $A_{u}$ reward for a gain of one unit of maximum flow equals $w_{u} \times \pi$.

In such a network game, each transportation-agent has to determine its individual strategy, i.e., the capacity $q_{i, j}$ of its own arcs, satisfying the constraints $q_{i, j} \in\left[\underline{q}_{i, j}, \bar{q}_{i, j}\right]$. The individual strategy of $A_{u}$ is denoted $Q_{u}=\left(q_{i, j}\right),(i, j) \in E_{u}$. It represents the vector of capacities chosen by $A_{u}$ for its arcs, with $\underline{Q} \leq Q_{u} \leq \bar{Q}$. A strategy $S$ in the network flow is the vector of individual strategies of all agents: 
$S=\left(Q_{1}, \ldots, Q_{m}\right)$.

The price paid by transportation-agent $A_{u}$ for its individual strategy $Q_{u}$ equals:

$$
P_{u}\left(Q_{u}\right)=C_{u} \times\left(Q_{u}-\underline{Q}_{u}\right)=\sum_{(i, j) \in E_{u}} c_{i, j} \times\left(q_{i, j}-\underline{q}_{i, j}\right)
$$

Given a strategy $S, F(S)$ denotes the maximum flow that can circulate on the network flow given the current values of capacities. For each $\operatorname{arc}(i, j)$, the circulating flow $f_{i, j}$ is such that $\underline{q}_{i, j} \leq f_{i, j} \leq q_{i, j}$. The maximum flow $F(S)$ is equal to the sum of flow circulating in the forward arcs of source node (i.e., $\left.F=\sum_{(s, j) \in E} f_{s, j}\right)$. Let us remark that $F(S)$ can be computed in polynomial time using the well-known Ford-Fulckerson algorithm ??Ford58]. We denote by $\underline{F}$ the maximum flow when capacities $q_{i, j}$ are set to $\underline{q}_{i, j}$ for all transportationagents (in other words, the largest possible flow at zero cost) and by $\bar{F}$ the maximum flow obtained when capacities $q_{i, j}$ are set to $\bar{q}_{i, j}$. Therefore, for any strategy $S$, it holds that $\underline{F} \leq F(S) \leq \bar{F}$.

With respect to the above payment scheme, the total reward given by the customer-agent for a circulating flow $F(S)$ under a strategy $S$ is $\pi \times(F(S)-\underline{F})$. The profit $Z_{u}(S)$ of transportation-agent $A_{u}$ under strategy $S$ is equal to the difference between its reward and spending:

$$
Z_{u}(S)=w_{u} \times \pi \times(F(S)-\underline{F})-P_{u}\left(Q_{u}\right)
$$

$Z(S)=\left(Z_{1}(S), \ldots, Z_{m}(S)\right)$ represents the overall profit vector.

The strategy profile $S_{-u}$ denotes the strategies of the $(m-1)$ agents, but agent $A_{u}$, that is $S_{-u}=\left(Q_{1}, Q_{2}, . ., Q_{u-1}, Q_{u+1}, . ., Q_{m}\right)$. Therefore, a strategy where only one agent $A_{u}$ modify its strategy is denoted by $S=\left(Q_{u}, S_{-u}\right)$ and the profit of agent $A_{u}$ resulting from such a strategy is denoted by $Z_{u}\left(Q_{u}, S_{-u}\right)$.

Example of a MA-MCMF Problem Let us consider a customer-agent willing to transport a flow of products from a given source node $A$ to a given sink node $D$. Two transportation-agents $A_{1}$ and $A_{2}$ are involved in the transportation process. The customer-agent gives a reward $\pi=120$ which is shared between agents following the sharing policy $w_{1}=w_{2}=\frac{1}{2}$. Fig. 1 displays the network topology. The set of arcs of each transportation-agent are $E_{1}=\{b=(A, C), c=$ $(B, C), d=(B, D)\}$ and $E_{2}=\{a=(A, B), e=(C, D)\}$, which are represented with plain and dotted arcs, respectively. Each arc in the graph of Fig. 3 is valuated by the interval of normal and maximum capacities $\left(\left[\underline{q}_{i, j}, \bar{q}_{i, j}\right]\right)$, and by the cost of increasing arc capacities $\left(c_{i, j}\right)$. For instance, arc $b$ of agent $A_{1}$ is valuated by the interval of capacities $[0,1]$ and the cost 30 . Transportationagent $A_{1}$ can choose to open the route $b$ from $A$ to $C$ with capacity $q_{A, C}=1$ and transportation-agent $A_{2}$ can choose to put the capacity of the route $e$ from $C$ to $D$ to capacity $q_{C, D}=1$. With this strategy, the maximum flow of product than can be transported $F(S)$ is equal to 1 (from $A$ to $C$ and from $C$ to $D$ ). 


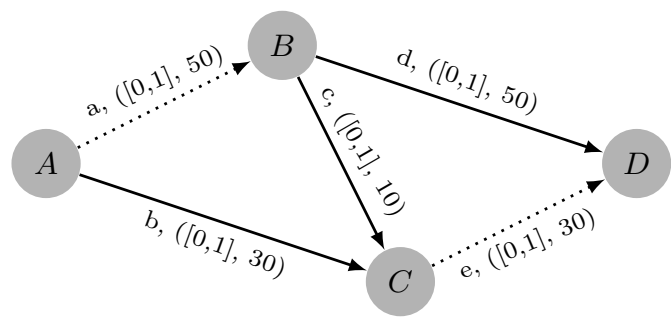

Fig. 1. Example of MA-MCMF problem

The total reward given by the customer-agent equals 120 and the rewards for $A_{1}$ and $A_{2}$ are equal to 60 . The cost for $A_{1}$ to open the route $b$ for one unit of flow is 30 and the cost for $A_{2}$ to open the route $e$ for one unit of flow is 30 too. Then, $Z_{1}=Z_{2}=60-30=30$, which means that this strategy is profitable for both agents.

\subsection{Mathematical Formulation}

Each agent having the objective of maximizing its own profit, the problem can be formalized as the following multi-objective mathematical program where $Z_{u}(S)$ is computed according to equation (2):

$$
\begin{aligned}
& \operatorname{Max}\left(F, Z_{1}(S), Z_{2}(S), \ldots, Z_{m}(S)\right) \\
& \text { s.t. } \\
& \begin{array}{ll}
\text { (i) } \quad f_{i, j} \leq q_{i, j}, \forall(i, j) \in E \\
\text { (ii) } \quad \sum_{(i, j) \in E} f_{i, j}-\sum_{(j, i) \in E} f_{j, i}=\left\{\begin{array}{l}
0 \forall i \neq s, t \\
F, i=s \\
-F, i=t
\end{array}\right. \\
\text { (iii) } \quad \underline{q}_{i, j} \leq q_{i, j} \leq \bar{q}_{i, j}, \forall(i, j) \in E \\
\quad f_{i, j} \geq 0, \forall(i, j) \in E
\end{array}
\end{aligned}
$$

The mathematical formulation (5) aims at finding an overall strategy $S$ that maximizes both the flow and the profit of all agents, each agent $A_{u}$ deciding the arc capacity $q_{i, j}, \forall(i, j) \in E_{u}$. Constraints $(i)$ represent the capacity constraints. Constraints (ii) impose the conservation of the flow.

\section{$3 \quad$ Efficiency vs. Stability}

A strategy is said efficient if it corresponds to a Pareto-optimal solution with respect to the above multi-objective program (5). The notion of Pareto optimality is concerned with social efficiency [21]. A Pareto strategy is preferred to any other strategy dominated by it.

Definition 1. Pareto optimal strategy: A strategy $S$ is Pareto-optimal if it is not dominated by any other strategy $S^{\prime}$. In other words, it does not exist any strategy $S^{\prime}$ such that $Z_{u}\left(S^{\prime}\right) \geq Z_{u}(S)$ for all $A_{u}$, with at least one inequality being strict. 
The set of Pareto optimal strategies is denoted by $S^{P}$.

On the other hand, a strategy is stable if there is no incentive for any agent to modify its decision in order to improve its profit. The stability of a strategy ensures that agents can trust each other. It is connected to the notion of a Nash equilibrium in non-cooperative game (see [23], [24] and [25]).

Definition 2. Nash Equilibrium strategy: given a sharing reward policy $w_{u}$, a strategy $S=\left(Q_{1}, \ldots, Q_{m}\right)$ is a Nash Equilibrium if for any agent $A_{u}$ with strategy $Q_{u}^{\prime}$, the following equation holds:

$$
Z_{u}\left(Q_{u}, S_{-u}\right) \geq Z_{u}\left(Q_{u}^{\prime}, S_{-u}\right), \quad \forall Q_{u}^{\prime} \neq Q_{u}
$$

We refer to $S^{N}$ as the set of Nash equilibria. Ideally, agents should choose a strategy which satisfy both Pareto optimality and Nash stability (i.e., $S \in S^{N} \cap S^{P}$ ). Nevertheless, since $S^{N} \cap S^{P}$ can be empty, such a strategy does not always exist. In this case, we are looking for a Nash equilibrium that is as efficient as possible with respect to the customer viewpoint. A Nash equilibrium that maximizes the flow circulating is indeed suitable both for maximizing the total reward and the customer satisfaction. The aim of this study is to find a stable strategy profile $S^{*}$ (i.e., a Nash Equilibrium) that maximizes the flow circulating. Let us also define the concept of a poor strategy. This concept will be useful for characterizing properly Nash equilibria.

Definition 3. Poor strategy: A strategy $S=\left(Q_{1}, \ldots, Q_{m}\right)$ with flow $F(S)$ is a poor strategy if and only if it exists an agent $A_{u}$ and an alternative strategy $Q_{u}^{\prime}$ such that $Z_{u}\left(S^{\prime}\right)>Z_{u}(S)$ and $F\left(S^{\prime}\right)=F(S)$, where $S^{\prime}=\left(Q_{u}^{\prime}, S_{-u}\right)$.

In other words, $S$ is a poor strategy if and only if one agent is able to increase its profit by changing unilaterally its strategy (modifying the capacity of some of its arcs), without modifying the overall flow in the network, nor the profits of other agents. It is obvious that for any poor strategy $S, S \notin S^{N} \cup S^{P}$.

A poor strategy $S=\left(Q_{u}, S_{-u}\right)$ can be easily transformed into a non-poor strategy $S^{\prime}=\left(Q_{1}^{\prime}, \ldots, Q_{m}^{\prime}\right)$ by proceeding to an adaptation of the strategy $Q_{u}$ of agent $A_{u}$ while keeping strategy defined by $S_{-u}$ fixed for the $m-1$ agents but agent $A_{u}$ such that $S_{-u}=\left(Q_{1}, Q_{2}, \ldots, Q_{u-1}, Q_{u+1}, \ldots, Q_{m}\right)$.

Given $F(S)=F\left(S^{\prime}\right)$ and $S_{-u}$, a non-poor strategy $S^{\prime}$ can be the solution of the following linear program:

$$
\begin{aligned}
& \operatorname{Max} \sum_{A_{u} \in \mathcal{A}} Z_{u}\left(S^{\prime}\right)=\sum_{A_{u} \in \mathcal{A}}\left[w_{u} \times \pi \times\left(F\left(S^{\prime}\right)-\underline{F}\right)-P_{u}\left(Q_{u}^{\prime}\right)\right] \\
& \text { s.t. } \\
& \text { (i) } Z_{u}\left(S^{\prime}\right)>Z_{u}(S), \forall A_{u} \in \mathcal{A} \\
& \text { (ii) } \quad F\left(S^{\prime}\right)=F(S)=\sum_{(s, j)} f_{s, j}^{\prime} \\
& \text { (iii) } f_{i, j}^{\prime} \leq q_{i, j}^{\prime}, \forall(i, j) \in E \\
& \text { (vi) } \sum_{(i, j) \in E} f_{i, j}^{\prime}-\sum_{(j, i) \in E} f_{j, i}^{\prime}=\left\{\begin{array}{l}
0 \forall i \neq s, t \\
F, i=s \\
-F, i=t
\end{array}\right. \\
& \text { (iv) } \underline{q}_{i, j} \leq q_{i, j}^{\prime} \leq \bar{q}_{i, j}, \forall(i, j) \in E_{u} \\
& f_{i, j}^{\prime} \geq 0, \forall(i, j) \in E
\end{aligned}
$$

The mathematical program (5) is used both to verify if a strategy is poor and to ameliorate the strategy in order to remedy to its poorness. For the former concern, if 
a solution to (5) exists and is different from $S$, then the strategy $S$ is poor. For the latter concern, the mathematical program (5) gives a non-poor strategy $S^{\prime}$ since it aims at maximizing the sum of profits of all the agents under the constraint that the flow remains constant and the profit of every agent in $S^{\prime}$ is at least greater or equal to the profit in $S$ (i.e., $\left.Z_{u}\left(S^{\prime}\right)>Z_{u}(S), \forall A_{u} \in \mathcal{A}\right)$. Therefore the following proposition holds.

Proposition 1. Any solution of the mathematical program (5) is non poor solution.

\section{Case analysis}

For sake of simplicity, it is assumed throughout this section, that $\underline{q}_{i, j}=0$. Therefore, the initial minimum circulating flow at zero cost is equal to $\underline{F}=0$.

\subsection{The Single-agent Case}

This section presents or recalls some basic properties related to classical network flow theory. In the single agent case (all the arcs belong to the same agent), a non-poor strategy $S$ for a given flow $F(S)$ is a strategy that minimizes the overall cost. Such minimization problem is well-identified in the literature as the minimum-cost maximum-flow problem [26].

Let us define, in the following section, how the total flow can be either increased or decreased, at minimum cost, using augmenting or decreasing paths. These notions will be used in section 4.2 .

Increasing the Max-flow. Given a flow $F(S)$ for strategy $S$, we are interested in increasing the flow value at minimum cost. For this purpose, we recall the well-known notion of an augmenting path, based on the concept of a residual graph $G_{f}(S)$, which is defined below.

Definition 4. Residual graph: Given a network $G=(V, E)$ and a flow $F(S)$, the corresponding residual graph $G_{f}(S)=\left(V, E_{r}\right)$ for a given strategy $S$ is defined as follows: each arc $(i, j) \in E$, having a maximum capacity $\bar{q}_{i, j}$ and a flow $f_{i, j}$ in $G$, is replaced by two arcs $(i, j)$ and $(j, i)$ in the residual graph. The arc $(i, j)$ has cost $c_{i, j}$ and residual capacity $r_{i, j}=\bar{q}_{i, j}-f_{i, j}$ and the arc $(j, i)$ has cost $c_{j, i}=-c_{i, j}$ and residual capacity $r_{j, i}=f_{i, j}$.

Definition 5. Augmenting path: An augmenting path is a path $P$ in $G_{f}(S)$ from the source $s$ to the sink $t$ through which the flow can be increased.

We refer to $\mathcal{P}$ as the set of augmenting paths. The greatest flow augmentation that can be achieved using $P \in \mathcal{P}$ is $r_{p}=\min \left\{r_{i j}:(i, j) \in P\right\}$.

An augmenting path in $G_{f}(S)$ is made of forward arcs (having the same direction in $G$ ) and backward arcs (having the opposite direction than the ones in $G$ ). The set of forward and backward arcs are denoted $P^{+}$and $P^{-}$, respectively.

The cost of augmenting the flow by one unit using the augmenting path $P \in \mathcal{P}$ is denoted $\operatorname{cost}(P)$. It is expressed as follows:

$$
\operatorname{cost}(P)=\sum_{(i, j) \in P^{+}} c_{i, j}-\sum_{(i, j) \in P^{-}} c_{i, j}
$$


Decreasing the Max-flow. When considering the problem of decreasing the flow at minimum cost in the network, we introduce the new concept of a decreasing path.

Definition 6. Decreasing path: a decreasing path $\bar{P}$ is a path in $G_{f}(S)$ from the sink node $t$ to the source node $s$ through which the flow can be decreased.

We refer to $\overline{\mathcal{P}}$ as the set of decreasing paths.

Similarly, a decreasing path in $G_{f}(S)$ is made of forward arcs (having the opposite direction than the one in $G_{f}(S)$ ) and backward arcs (having the same direction in $G_{f}(S)$ ). The set of forward and backward arcs are denoted $\bar{P}^{+}$and $\bar{P}^{-}$, respectively. The profit of decreasing the flow along $\bar{P}$ can be expressed as follows:

$$
\operatorname{profit}(\bar{P})=\sum_{(i, j) \in \bar{P}^{+}} c_{i, j}-\sum_{(i, j) \in \bar{P}^{-}} c_{i, j}
$$

Example Consider the network flow $G(V, E)$, displayed in Fig. 2, composed of seven $\operatorname{arcs} E=\{a, b, c, d, e, f, g\}$. The set of vertex is $V=\{s, 1,2,3,4, t\}$. Each arc in the graph is valuated by the interval of normal and maximum capacities, and by the cost of increasing arc capacities $\left(\left[\underline{q}_{i, j}, \bar{q}_{i, j}\right], c_{i, j}\right)$, where $\left[\underline{q}_{i, j}, \bar{q}_{i, j}\right]=[1,20], \forall(i, j) \in E$. For instance, arc $(s, 1)$ from node $s$ to node 1 is valuated by the capacity interval $[1,20]$ and the cost 100 .

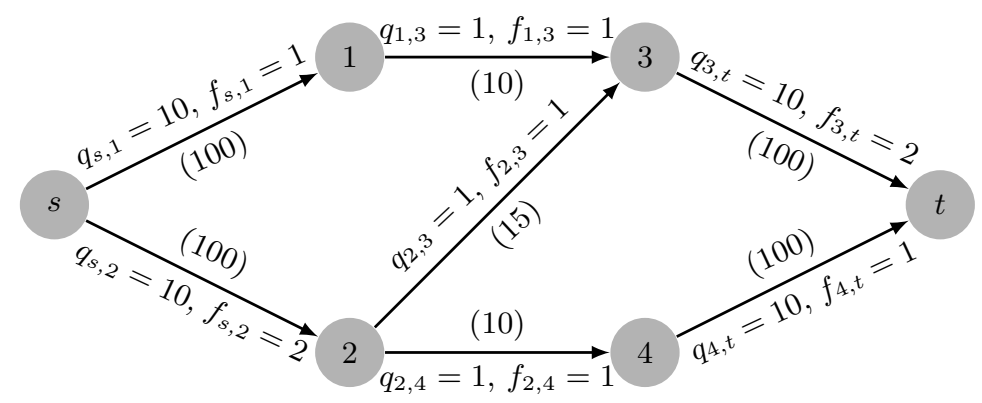

(a) Strategy $S_{0}$

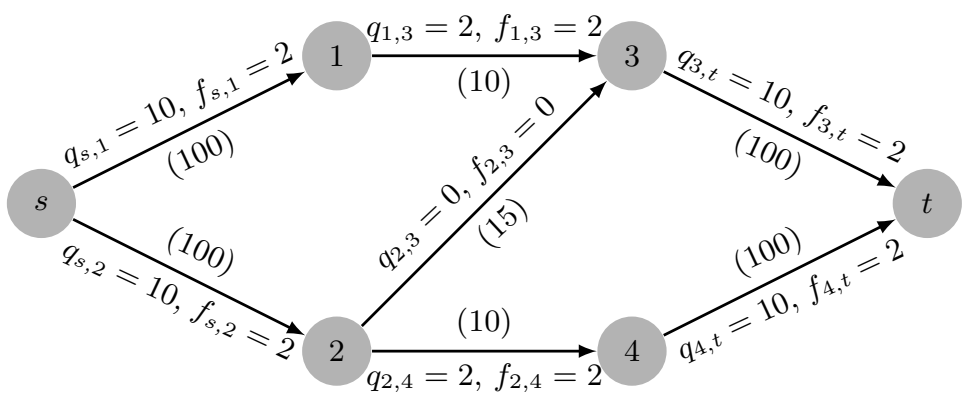

(b) strategy $S_{1}$

Fig. 2. Example of single-agent network flow 
The initial strategy $S_{0}$ is described in Fig. 2(a) with flow equal to $F\left(S_{0}\right)=3$. The best way to increase the flow in the network is to use the augmenting path having minimum cost, (i.e., $P=s-1-3-2-4-t$ ), to increase the flow on forward arcs by one unit and decrease the flow on backward arcs by one unit. With the obtained strategy $S_{1}=(2,2,2,0,2,2,2)$ (see Figure 2(b)), the maximum flow of product that can be transported $F\left(S_{1}\right)$ is equal to 4 and the cost incurred by the flow increase throughout the augmenting path $P$ is equal to $\operatorname{cost}(P)=5$.

\subsection{The Multi-agent Case}

In the multi-agent context, any agent $A_{u}$ can decrease (or increase) unilaterally its arc capacities to improve its profit $Z_{u}$. In this context, we introduce the concept of profitability of an augmenting or a decreasing path and provide a characterization of a Nash equilibrium strategy for the MA-MCMF problem.

Increasing the Max-flow. Let us introduce the notion of a profitable augmenting path. In the multi-agent context, an augmenting path is composed by a set of forward and backward arcs $P=\left\{P^{+}, P^{-}\right\}$such that by simultaneously increasing $q_{i, j}$ increased by one unit $\forall(i, j) \in P^{+}$and decreasing by one unit $\forall(i, j) \in P^{-}$, it is possible to increase the overall flow by one unit.

The cost of an augmenting path for agent $A_{u}, \operatorname{cost}_{u}(P)$ is expressed as follows:

$$
\operatorname{cost}_{u}(P)=\sum_{(i, j) \in P^{+} \cap E_{u}} c_{i, j}-\sum_{(i, j) \in P^{-} \cap E_{u}} c_{i, j}
$$

Definition 7. Profitable augmenting path. An augmenting path $P \in \mathcal{P}$ is said profitable for all agents if, for every agent $A_{u}$ involved in $P, \operatorname{cost}_{u}(P)<w_{u} \times \pi$.

This means that through a profitable augmenting path, increasing the flow by one unit, is profitable for all the agents owning the arcs of the path (i.e., the profit of an agent $A_{u}$ for increasing the flow by one unit verify $Z_{u}(S)=w_{u} \times \pi-\operatorname{cost}_{u}(P)>0$, where $\operatorname{cost}_{u}(P)$ is the reduced cost).

Decreasing the Max-flow. Now, the notion of profitability is introduced. In the multi-agent context, a decreasing path $\bar{P}=\left\{\bar{P}^{+}, \bar{P}^{-}\right\}$is composed of forward and backward arcs. If $q_{i, j}$ is decreased by one unit, $\forall(i, j) \in \bar{P}^{+}$, and increased by one unit, $\forall(i, j) \in \bar{P}^{-}$, the overall flow is decreased by one unit.

Considering an agent $A_{u}$, the profit $\operatorname{profit}_{u}(\bar{P})$ generated by decreasing capacity by one unit through a decreasing path is defined as follows:

$$
\operatorname{profit}_{u}(\bar{P})=\sum_{(i, j) \in \bar{P}^{+} \cap E_{u}} c_{i, j}-\sum_{(i, j) \in \bar{P}^{-} \cap E_{u}} c_{i, j}
$$

Definition 8. Profitable decreasing path. A decreasing path $\bar{P} \in \overline{\mathcal{P}}$ is profitable if there is one agent $A_{u}$ such that profit $(\bar{P})>w_{u} \times \pi$.

In other words, through a profitable decreasing path, decreasing the flow by one unit is profitable for one agent, to the detriments of the others.

In the multi-agent context, it is important to characterize strategies in which some agents can decrease or increase the overall flow. Therefore, it is important to find profitable augmenting paths in order to increase flow without generating decreasing paths that are profitable for some agent, hence preserving stability. 
Proposition 2. Nash Equilibrium.

For a given non-poor strategy profile $S, S$ is a Nash Equilibrium if and only if:

- $\forall A_{u} \in \mathcal{A}, \forall P \in \mathcal{P}$ such that $(i, j) \in E_{u}$

$$
\operatorname{cost}_{u}(P)>w_{u} \times \pi
$$

$-\forall A_{u} \in \mathcal{A}, \forall \bar{P} \in \overline{\mathcal{P}}$

$$
\operatorname{profit}_{u}(\bar{P}) \leq w_{u} \times \pi
$$

Proof. Consider a strategy $S$ and a transportation-agent $A_{u}$. If $S$ is poor, then $S$ is not a Nash equilibrium. If $S$ is non poor, $A_{u}$ can only improve its situation by increasing or decreasing the flow. In the former case, for an additional unit of flow, $A_{u}$ receives $w_{u} \times \pi$. Such a flow increase is profitable to $A_{u}$ if and only if there is an augmenting path $P$ such that $\operatorname{cost}_{u}(P)<w_{u} \times \pi$, which contradicts equation (10). In the latter case, vice-versa, decreasing the flow by one unit is profitable if and only if there exists a decreasing path $\bar{P}$ such that $\operatorname{profit}_{u}(\bar{P})>w_{u} \times \pi$, which contradicts equation (11). Therefore, if and only if for no agent any of those conditions holds, no agent $A_{u}$ can individually improve its profit, and $S$ is a Nash equilibrium.

Example Let us come back to the first example (cf. section 2.1) to illustrate the notions of augmenting and decreasing path in the multi-agent case.

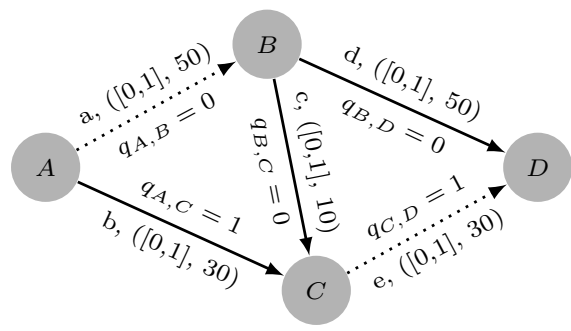

(a) Strategy $S_{1}$

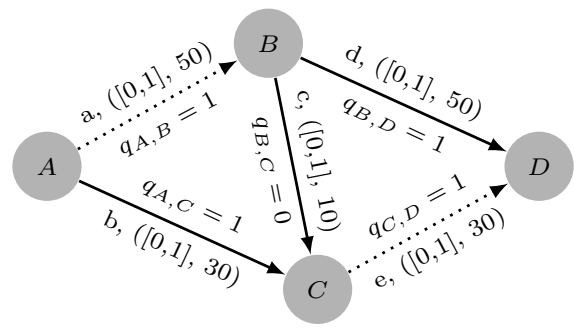

(b) Strategy $S_{2}$

Fig. 3. Example of multi-agent network flow

Consider an initial flow on the network equal to its minimum value $\underline{F}=0$ corresponding to an initial strategy $S_{0}$. Increasing the flow is possible throughout the profitable augmenting path $P=(A-C-D)$, which leads to the strategy $S_{1}=(0,1,0,0,1)$ (see Figure 3(a)) with $F\left(S_{1}\right)=1$ and $Z_{1}\left(S_{1}\right)=Z_{2}\left(S_{1}\right)=60-30=30$ where the part of shared reward is $w_{u} \times \pi=60$ and the cost of the path $P$ is $\operatorname{cost}_{u}(P)=30$ for both agents. From this strategy, the flow can be increased along the profitable augmenting path $P^{\prime}(=A-B-D)$, leading to the strategy $S_{2}=(1,1,0,1,1)$ (see Figure $3(\mathrm{~b})$ ) with $F\left(S_{2}\right)=2$. The cost of the augmenting path for every agent is equal to $\operatorname{cost}_{u}\left(P^{\prime}\right)=50$ and the part of the shared reward for the additional unit of flow is equal to $w_{u} \times \pi=60$. Therefore, the profit of both agents is equal to $Z_{u}\left(S_{2}\right)=Z_{u}\left(S_{1}\right)+(60-50)=30+10=40$.

Note that, for the strategy $S_{2}$, there exists a profitable decreasing path $P "=(D-$ $B-C-A)$ from sink node $D$ to source node $A$ which is profitable for agent $A_{1}$. 
In fact, $A_{1}$ can improve its own profit, by decreasing back the flow on $b$ and $d$ by one unit and increasing the flow on arc $c$ by one unit. This leads to the strategy $S_{3}=(1,0,1,0,1)$ (see Figure 4 ) with $F\left(S_{3}\right)=1$ and profits $Z_{1}\left(S_{3}\right)=60-10=50$ and $Z_{2}\left(S_{3}\right)=60-(50+30)=-20$, which is obviously bad for $A_{2}$. Therefore, although the strategy $S_{2}$ corresponds to a Pareto Optimum, which leads to a maximization of agent's profits, it is not a stable strategy. Strategy $S_{1}$ is a Nash Equilibrium but not Pareto Optimum. Therefore, in our example there is no a strategy which is both in $S^{N}$ and $S^{P}$. The motivation of this paper is to search for a Nash-stable solution which is as efficient as possible, i.e., which maximizes $F(S)$.

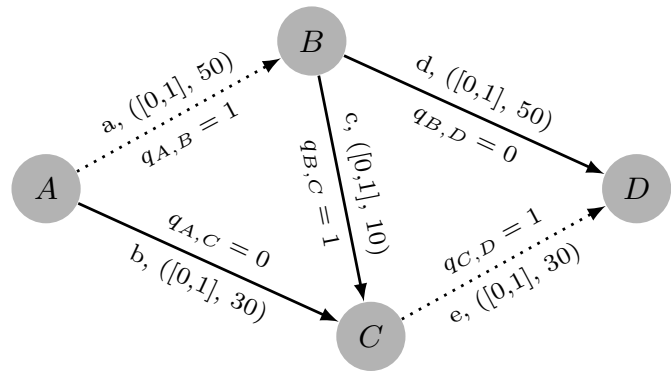

Fig. 4. Strategy $S_{3}$

\subsection{The Special Case $\left|E_{u}\right|=1, \forall A_{u}$}

In this section, we consider the special multi-agent case where each arc is managed by a specific agent. For this case, we show that finding a Nash equilibrium that maximizes the flow can be done in polynomial time. For sake of simplicity, we denote by $u$ the unique arc of the agent $A_{u}$. In this context, increasing the flow by one unit brings to the agent $A_{u}$ the reward $w_{u} \times \pi$ and since an agent manages only a single arc $u$ then it is easy to compare the reward with the cost of increasing arc's capacity $c_{u}$ of arc $u$. It is possible to divide the set of agents $\mathcal{A}$ into two subsets $\mathcal{A}^{+}$and $\mathcal{A}^{-}$as follows:

$$
\begin{aligned}
& \mathcal{A}^{+}=\left\{A_{u}, 1 \leq u \leq m, / c_{u}<w_{u} \times \pi\right\} \\
& \mathcal{A}^{-}=\left\{A_{u}, 1 \leq u \leq m, / c_{u} \geq w_{u} \times \pi\right\}
\end{aligned}
$$

Signification of each group of agents:

On the one hand, for any agent belonging to the group $\mathcal{A}^{+}$, it is profitable to increase the capacity of its arc (i.e., $w_{u} \times \pi-c_{u}>0$ ) if it increases the overall flow in the network (i.e., its arcs belong to an augmenting path). On the other hand, it is not profitable for any agent belonging to $\mathcal{A}^{-}$to increase its arc capacity since $w_{u} \times \pi-c_{u} \leq 0$.

Consider the initial strategy $S=\left(Q_{1}, \ldots, Q_{m}\right)$ defined by:

$$
\begin{aligned}
& Q_{u}=\left(\bar{q}_{u}\right), \forall A_{u} \in \mathcal{A}^{+} \\
& Q_{u}=\left(\underline{q}_{u}\right), \forall A_{u} \in \mathcal{A}^{-}
\end{aligned}
$$


We highlight that the strategy $S$ can be poor since some arcs of the agents belonging to $\mathcal{A}^{+}$can have an opened capacity greater than the value of the flow traversing them (i.e., $\left.f_{i, j}(S)<q_{i, j}(S)\right)$. Nevertheless, using LP formulation (5), finding a non-poor strategy $\hat{S}$ starting from $S$ is easy. This leads to a non-poor strategy $\hat{S}$ with the same value of flow $F(\hat{S})=F(S)$.

Notice that $\hat{S}$ may not be unique, since different non-poor strategies can be obtained. We are going to prove now the following property:

Proposition 3. Strategy $\hat{S}$ is a Nash Equilibrium, and there is no Nash Equilibrium with greatest flow.

Proof. This proof is organized in two parts:

- Proof that $\hat{S}$ is a Nash Equilibrium:

Let us consider the arcs of $A_{u} \in \mathcal{A}^{-}$. Since their capacities are at their minimum value, $F(\hat{S})$ can be increased only by increasing the capacities throughout an augmenting path. Since for agents in $\mathcal{A}^{-}, c_{u}>w_{u} \times \pi$, then no agent in $\mathcal{A}^{-}$has any incentive to increase its arc capacity.

Now let consider the agents $A_{u} \in \mathcal{A}^{+}$. If, in $\hat{S}, q_{u}=\bar{q}_{u}$ then $A_{u}$ can improve its situation only by decreasing its arc capacity throughout a decreasing path. Since for agents in $\mathcal{A}^{+}, c_{u} \leq w_{u} \times \pi$, no agent $A_{u}$ can take profit from decreasing back its arc capacity. If in $\hat{S}, q_{u}<\bar{q}_{u}$, one agent $A_{u}$ might increase its arc capacity throughout an augmenting path such that all forward arcs belong to him (else it is not possible to increase the value of the flow). Since each agent owns exactly one arc, such a situation cannot occur.

Finally, since no agent is able to improve its situation by itself, $\hat{S}$ is a Nash equilibrium.

- Proof that $\hat{S}$ is the best Nash Equilibrium:

Suppose that there is a strategy $S^{\prime}$ such that $F\left(S^{\prime}\right)>F(\hat{S})$. This strategy requires that the capacity of at least one arc of $A_{u} \in \mathcal{A}^{-}$has to be increased with respect to strategy $\hat{S}$. But since $c_{u}>w_{u} \times \pi, \forall A_{u} \in \mathcal{A}^{-}, S^{\prime}$ is not a Nash Equilibrium (see proposition (2)).

\section{Problem Complexity}

In this section, we discuss the complexity of finding a Nash equilibrium that maximizes the flow in the network.

\subsection{Finding a feasible solution}

Firstly, let us discuss the complexity of a simplified version of the considered problem in which we substitute the Nash Equilibrium constraint by a looser constraint stating that the profit of all agents has to be non-negative, i.e., $Z_{u}(S) \geq 0, \forall A_{u} \in \mathcal{A}$.

Proposition 4. The multi-agent Min-Cost Max-Flow problem which aims at maximizing $F(S)$ under the constraints that agents have non-negative profits $Z_{u}(S) \geq 0$, with $q_{i, j} \in \mathbb{R}^{+}$, can be solved in polynomial time. 
Proof. This problem can be solved by the following linear mathematical problem where constraints (iii) impose that the profit of all agents has to be positive or null:

$$
\begin{aligned}
& \operatorname{Max} F=\sum_{(s, j) \in E} f_{s, j} \\
& \text { s.t. } \\
& \text { (i) } f_{i, j} \leq q_{i, j}, \forall(i, j) \in E \\
& \text { (ii) } \quad \sum_{(i, j) \in E} f_{i, j}-\sum_{(i, j) \in E} f_{j, i}=\left\{\begin{array}{l}
0 \forall i \neq s, t \\
F, i=s \\
-F, i=t
\end{array}\right. \\
& \text { (iii) } w_{u} \times \pi \times(F-\underline{F})-\sum_{(i, j) \in E_{u}} c_{i, j} \times\left(q_{i, j}-\underline{q}_{i, j}\right) \geq 0, \forall A_{u} \in \mathcal{A} \\
& \text { (iv) } \underline{q}_{i, j} \leq q_{i, j} \leq \bar{q}_{i, j}, \forall(i, j) \in E \\
& f_{i, j} \geq 0, \forall(i, j) \in E
\end{aligned}
$$

Therefore, this problem can be solved using linear programming in polynomial time.

\subsection{Finding a Nash Equilibrium with Bounded Flow}

We now consider the decision problem to determine if there exists a strategy which is a Nash equilibrium, with a flow greater than a given value. This problem can be defined as follows:

\section{Nash-Equilibrium Bounded Flow (NEBF).}

Given a tuple $\langle G, \mathcal{A}, Q, \bar{Q}, C, \pi, W>$ as defined in section 2 and an integer $\varphi$, is it possible to find a Nash Equilibrium strategy profile $S$ such that $F(S)>\varphi$ ?

Proposition 5. Problem NEBF is strongly NP-complete.

Proof. The NP-completeness of this problem can be proved using a reduction from the well-known 3-partition problem, which is known to be NP-complete in the strong sense [27]. First, MA-MCMF is in NP since, given a strategy $S, F(S)$ can be determined

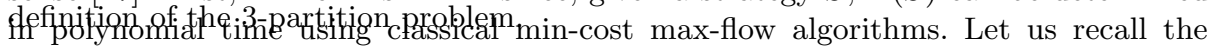

3-partition. Given a set $\zeta=\left\{a_{0}, \ldots, a_{K-1}\right\}$ of $K=3 k$ positive integers, such that $\sum_{l=0}^{K-1} a_{l}=k \times B$ and $\left.\left.a_{l} \in\right] B / 4, B / 2\right]$, is it possible to partition $\zeta$ into $k$ subsets so that the sum of integers in each subset is equal to $B$ ?

An instance of the MA-MCMF problem with controllable capacities can be generated from an arbitrary instance of the 3-partition problem as follows.

From the 3-partition problem instance, we build up a network $G$ with $k \times K$ arcs and $K+1$ nodes where the first one is source node $V_{0}=s$ and the last one is the sink node $V_{K}=t$. An agent $A_{u} \in \mathcal{A}=\left\{A_{1}, \ldots, A_{k}\right\}$ owns $K$ arcs.

The tail of an $\operatorname{arc} e_{i}$ is $V_{i \operatorname{div} K}$, its head is $V_{(i \operatorname{div} K)+1}$. Between nodes $V_{i \operatorname{div} K}$ and $V_{i \text { div } K+1}$, there are $k$ parallel arcs, indexed from $i$ to $(i+K)$ step $k$, each of them belonging to a specific agent: arc $e_{i}$ belongs to $A_{i \operatorname{div} K}$. The cost of $\operatorname{arc} e_{i}$ is $c_{e_{i}}=a_{i \bmod K}$. In other words, to any positive integer $a_{l} \in \zeta$ is associated $k$ parallel arcs with, same head and tail, maximum capacity $\bar{q}_{e_{i}}=1$ and cost $a_{l}$. The total reward is set to $\pi=(B+\epsilon) k, \epsilon$ being an arbitrary small positive value. The sharing policy is defined by $w_{u}=1 / k$. Therefore, agent's unit reward is $w_{u} \pi=B+\epsilon$, identical for all agents. The objective is to determine whether it exists a Nash strategy such that $F(S)>0$ ? 
For illustration, the resulting network flow obtained from the 3-partition instance defined by $k=3, \zeta=\{7,8,7,7,7,8,9,10,9\}$ and $B=24$. We have $k=3$ agents and $K * k=27$ arcs is displayed in Figure 5. Between nodes $i$ and $i+1$, we find $k=3$ arcs with cost $a_{i+1}$. The problem is to find, whether it exists, a Nash strategy such that the flow is strictly greater than 0 . In that example, using the augmenting path with bold arcs allows to obtain a one-unit total flow, which is a Nash equilibrium since every agent does not pay more than its part of reward $\left(w_{u} \pi=B+\epsilon=24+\epsilon\right)$. But we remark that, any equivalent stable path is also a solution to the original 3-Partition problem.

Let us prove this last property in a general way. Consider the strategy $\underline{S}$ where all arcs have normal capacity, $q_{i, j}=0$. The resulting flow obviously equals to $F(\underline{S})=0$. With respect to $\underline{S}$, we observe that an agent can increase the flow by the amount $\delta \in] 0,1]$, increasing the capacities of all its arcs by the same amount $\delta$. However, doing so, the agent pays $k B \delta$ and only gains $(B+\epsilon) \delta$. Hence, the new strategy is not profitable and cannot be a Nash equilibrium. In order to obtain a Nash equilibrium, the total cost incurred by each agent for increasing its arc capacities must not exceed $B$, otherwise at least one agent will be interested in decreasing back its capacities (i.e., the residual graph cotains a profitable decreasing path).

Due to the topology of the network, in order to increase the flow, exactly $K=3 k$ arcs must be involved in an augmenting path. In any Nash equilibrium strategy with flow strictly greater than 0 , the augmenting path having to be profitable for every agent, it must be made of exactly three arcs per agent. The total cost for every agent equals exactly $B$.

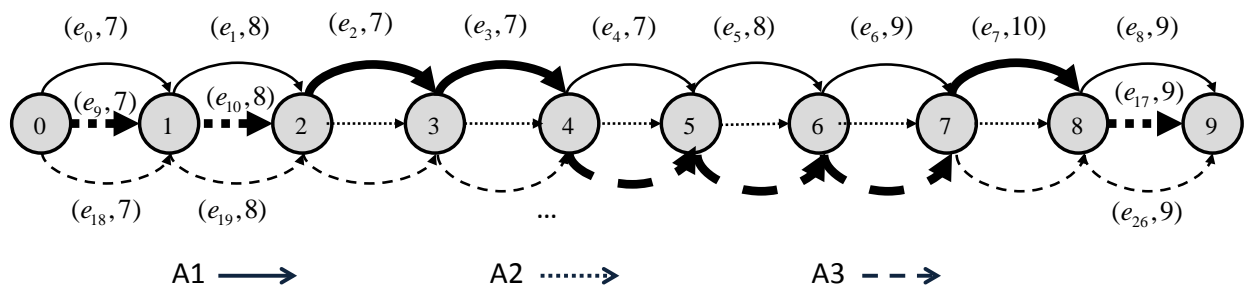

Fig. 5. Reduction from 3-PARTITION problem with $k=3$

\section{Conclusions}

This paper presents a new game theory framework for a multi-agent network flow problem with controllable capacities. We consider that a final customer gives a reward, shared among agent, for any additional unit of flow circulating in the network. Each agent has the possibility to modify the capacities of its arcs at a given cost. We particularly point out the notions of efficiency and stability of a strategy and we introduce the notion of profitable augmenting or decreasing paths. We also prove that finding a Nash Equilibrium strategy with maximum flow is NP-hard in the strong sense. 
Further works are ongoing to propose a linear mathematical model to find a Nash Equilibrium. Distributed heuristic able to find a Nash equilibrium are also under study.

\section{Acknowledgments}

This work was supported by the ANR project no. ANR-13-BS02-0006-01 named Athena.

\section{References}

1. B. Chen and H.H. Cheng: A Review of the Applications of Agent Technology in Traffic and Transportation Systems. IEEE transactions of intelligent transportation systems. vol. 11(2), pp. 485-497 (2010)

2. M. Pechoucek and V. Marik: Industrial deployment of multi-agent technologies: review and selected case studies. Autonomous Agents and Multi-Agent Systems. Vol. 17 (3), pp. 397-431 (2008)

3. C. Briand and A. Agnetis and J.C. Billaut: The multiagent project scheduling problem : complexity of finding an optimal Nash equilibrium. Proceedings of PMS conference, , pp. 106-109. (2012)

4. F. Zhang and R. Roundy and M.C. Akanyildrim and W.T. Huh: Optimal capacity expansion for multi-product, multi-machine manufacturing systems with stochastic demand. IIE Transactions. vol. 36(1), pp. 23-26 (2004)

5. S. Malcolm and S. Zenios: Robust optimization for power systems capacity expansion under uncertainty. Journal of the Operational Research Society. vol. 45(9), pp. 1040-1049 (1994)

6. M. Laguna: Applying robust optimization to capacity expansion of one location in telecommunications with demand uncertainty. Management Science. vol. 44(11), pp. 101-110 (1998)

7. V.N. Hsu: Dynamic capacity expansion problem with deferred expansion and agedependent shortage cost. Management Science. vol. 4(1), pp. 44-54 (2002)

8. T.Magnanti and R. Wong: Network design and transportation planning: Models and algorithms. Transportation Science. vol. 18(1), pp. 1-55 (1984)

9. D.R. Fulkerson: Increasing the Capacity of a Network: The Parametric Budget Problem. Management Science. vol. 5(4), pp. 472-483 (1959)

10. F. Ordonez and J. Zhao: Robust Capacity Expansion of Network flow. Networks. vol. 50(2), pp. 136-145. Wiley-Interscience (2007)

11. E. Tardos and T. Wexler: Network formation games and the potential function method. Algorithmic Game Theory. pp. 487-516, Cambridge University Press (2007)

12. R. Krzysztof Apt and E. Markakis: Diffusion in Social Networks with Competing Products. Proceedings of the 4th international conference on Algorithmic game theory, pp. 212-223. IEEE Press, Springer-Verlag (2011)

13. E. Resnick and Y. Bachrach and R. Meir and J.S. Rosenschein: The Cost of Stability in Network Flow Games. LNCS. vol. 5734, pp 636-650 (2009)

14. R. Evaristo and P.C. Van Fenema: A typology of project management: emergence and evolution of new forms. International Journal of Project Management. vol. 17(5), pp. 275-281 (1999)

15. P. De and E.J. Dunne and J.B. Ghosh and C.E. Wells: Complexity of the Discrete Time-Cost Tradeoff Problem for Project Networks. European Journal of Operations Research, 45(2), pp. 302-306 (1997) 
16. M.A. Estevez Fernandez: A game theoretical approach to sharing penalties and rewards in projects. European Journal of Operational Research. vol. 216(3), pp. 647-657 (2012)

17. A. Agnetis and C. Briand and J-C. Billaut and P. Sucha: Nash equilibria for the multi-agent project scheduling problem with controllable processing times. Under revision Journal of Scheduling (2014).

18. J. Kamburowski: On the minimum cost project schedule. International Journal of Management Science. vol. 22(4), pp. 401-407. (1994)

19. C. Briand and P. Sucha and S.U. Ngueveu: Finding an optimal Nash equilibrium to the multi-agent project scheduling problem. Submitted to Journal of Operation Research.

20. G.P. Cachon and M.A. Lariviere: Supply Chain Coordination with RevenueSharing Contracts: Strengths and Limitations. International Journal of Management Science. vol. 51(1), pp. 30-44. (2005)

21. M. Ehrgott: Multicriteria Optimization. Algorithmic Game Theory. pp. 487-516, Springer (2005)

22. L.R. Ford and D.R. Fulkerson: Flow in Networks. Princeton University Press, New Jersey (1958)

23. J. Nash: Equilibrium points in n-person games. Proceedings of the National Academy of Sciences. Vol. 36(1), pp. 48-49 (1950).

24. J. Nash: Non-Cooperative Games. The Annals of Mathematics. Vol. 54(2), pp. 286-295 (1951).

25. Y. Shoham and K. Leyton-Brown: Multiagent Systems: Algorithmic, GameTheoretic, and Logical Foundations. New York: Cambridge University Press, ISBN 978-0-521-89943-7 (2009).

26. R.G. Busacker and P.J. Gowen: A Procedure for Determining a Family of MinimalCost Network Flow Patterns. Defense Technical Information Center. Edition 15 (1961)

27. M.R. Garey and D.S. Johnson: Computers and Intractability: A Guide to the Theory of NP-Completeness. W.H. Freeman \& Co. New York, USA (1979) 